\title{
Safari to masking land: A hunt for the elusive $U^{1}$
}

\author{
CHARLES W. ERIKSEN, ${ }^{2}$ BARBARA B. BECKER, AND JAMES E. HOFFMAN \\ UNIVERSITY OF ILLINOIS
}

Due to the theoretical interest in whether $U$-shaped visual masking functions are obtained when discrimination responses are required of the $S$ s, an attempt was made to replicate the Weisstein and Haber (1965) experiment. The attempt was unsuccessful. The results indicated that masking was uniform over a temporal range of approximately $50 \mathrm{msec}$. The possibility of individual differences in the shape of masking functions was investigated in two subsequent experiments with negative results. Overall, the results of the three experiments supported a temporal integration account of masking when assessed by detection or discrimination criteria. Some possible reasons for discrepancies with the Weisstein and Haber (1965) experiment were considered.

When one visual stimulus is followed by a second within a range of approximately 0 to $100 \mathrm{msec}$, the second stimulus often "masks" the first one. This may cause distortion of the first stimulus, making it impossible to identify, or the first stimulus may appear dimmer, seem to move, or seem to disappear. Depending upon the nature of the stimulus, the mask and the judgment required of $S$, the masking effect may decrease monotonically as the time or interstimulus interval (ISI) between target and mask increases, or the masking effect may be greatest at some delay time between 0 and $100 \mathrm{msec}$, yielding a U-shaped function (see Kahneman, 1968, for review of the literature on masking phenomena).

The most general and perhaps the most simple explanation of masking phenomena is that stimuli separated by temporal intervals of less than $100 \mathrm{msec}$ sum together and are perceived as a composite much as though they had been simultaneously presented. The best known example of this summation process in vision is Bloch's law, which refers to the frequently confirmed finding that the duration and the intensity of light have a reciprocal relation over a temporal interval referred to as the critical duration. Within the critical duration, the energy of the stimulus is completely summed. This critical duration has been reported to be as long as $100 \mathrm{msec}$ and even longer in the perception of form (Kahneman \& Norman, 1964). The period or duration of complete summation is followed by another period in which summation is inversely proportional to temporal duration, and eventually performance becomes solely dependent upon intensity. The durations over which complete and partial summation are obtained depend in part upon the task and in part upon whether the stimulation is continuous or interrupted (Schurman, 1970). However, for the most part, these durations are in the range of the temporal durations over which masking effects are obtained.

An explanation of masking phenomena in terms of lack of fine temporal resolution in the visual system then requires that the next level of explanation address itself to why the target stimulus is more difficult to perceive or identify when it is a composite with the masking stimulus and to an understanding of the temporal integration process.

Under circumstances where the luminances of the target, mask, and interstimulus fields are unequal, masking can be understood as a reduction in effective contrast of the target due to the summation of luminances across the three fields (Eriksen, 1966). Under other circumstances where luminance summation-contrast reduction effects are controlled, impairment in target identification would appear to be attributable to inhibitory effects (Flom, Weymouth, \& Kahneman, 1963) or perhaps to changes in the acuity requirements for target identification when the target becomes a composite with the masking stimulus.

An explanation of masking as due to the target and masking stimuli summing together into a composite would seem to require that the maximum impairment in target identification would occur when the mask was presented simultaneously with the target. Most masking experiments that have used an identification criterion have indeed found that masking is a decreasing monotonic function of ISI. Several of the exceptions to the general findings have not been successfully replicated (Eriksen \& Marshall, 1969; Schurman \& Eriksen, 1969).

Some confusion as to the form of the masking function has occurred due to the use of different judgmental responses employed by different investigators. The $S$, for example, can be asked to detect the presence or absence of the target, to identify the target from among several alternatives, or to judge the apparent b rightness or clarity of the target. Detection or discrimination responses have generally led to monotonic masking functions, whereas judgments as to the apparent brightness of the target typically yield a U-shaped function where the apparent brightness of the target decreases from simultaneous presentation of target and mask to some minimal value at an ISI before again increasing to normal brightness (Alpern, 1953). This type of masking has at times been called metacontrast and has been restricted to those cases involving adjacent stimuli of equal energy (Fehrer \& Smith, 1962; Stigler, 1910; Weisstein, 1968). In other works, Weisstein has extended metacontrast to include not only judgment of apparent brightness of the target but also the detection of the target. However, Schiller and Smith (1966) have presented evidence to show that when the response required of $S$ is a detection of the target, monotonic masking functions are attained, whereas the U-shaped functions are characteristic of judgments of apparent brightness. This position would seem to require that the phenomenal dimming, which is readily observed at ISIs in the region of $80 \mathrm{msec}$, does not impair the S's ability to detect or identify the target. In other words, the phenomenal dimming may be mediated by a process independent of that providing the information for detection or identification. Eriksen and Colgate (1970), for example, have recently shown that the identifiability of forms is independent of apparent motion.

An important exception to the above conclusions is an experiment by Weisstein and Haber (1965) in which a U-shaped masking function was obtained where the $S$ was required to discriminate whether the target was the letter $\mathrm{D}$ or $\mathrm{O}$. The results of this experiment were confirmed by Mayzner's students in a class project (Mayzner, Blatt, Buchsbaum, Friedel, Goodwin, Kanon, Keleman, \& Nilsson, 1965). However, Schiller has been unsuccessful in several attempts to replicate the findings. ${ }^{3}$ In view of the theoretical importance of the issue, the present experiments were undertaken. The first experiment was as close a replication of the original Weisstein and Haber study (1965) as we were able to carry out. The 
subsequent two experiments were follow-ups on the results of this replication.

\section{Method}

\section{EXPERIMENT 1}

Subjects. The senior author and three female undergraduate students at the University of Illinois were the Ss in the experiment. The undergraduates were paid volunteers.

Apparatus and stimuli. A Scientific Prototype Model GA three-field tachistoscope was used in which the lamps were replaced with Sylvania F4T5/CWX bulbs. All fields were maintained at a luminance of $9.5 \mathrm{~mL}$. The stimuli were the capital letters $O$ and $D$. They were drawn with black India ink on white plastic cards using a Leroy lettering set, template No. $500 \mathrm{CL}$, and a No. 6 point. Masking rings were drawn on transparent plastic cards with a compass and a No. 5 point. At the viewing distance of 48 in., the letter $O$ subtended $.62 \mathrm{deg}$ of visual angle vertically by .50 deg visual angle horizontally and the letter $D$ subtended .62 deg vertically by $.45 \mathrm{deg}$ horizontally. Thickness of the letters was $.09 \mathrm{deg}$. The masking rings had an inner diameter of .62 deg and a thickness of $.07 \mathrm{deg}$. Weisstein and Haber (1965) give the dimensions of their letters as $.62 \mathrm{deg}$ vertically by $.30 \mathrm{deg}$ horizontally, with $.61 \mathrm{deg}$ for the inner diameter of the masking ring. Letters had a thickness of $.08 \mathrm{deg}$ and rings a thickness of .05 deg. Weisstein and Haber (1965) state that the angular separation between the outer edge of the letter and the inner edge of the ring was $.083 \mathrm{deg}$. This would appear to be some kind of an average since their figures indicate that the letters and rings would overlap at the top and bottom and maximum angular separation at the sides would be $.155 \mathrm{deg}$. In the present experiment, the angular separation varies from $0 \mathrm{deg}$ at the top and bottom for both letters to a maximum at the sides of $.06 \mathrm{deg}$ for the 0 and $.085 \mathrm{deg}$ for the D.

As in the Weisstein and Haber experiment (1965) the letters and their accompanying masks were presented in four positions, two to the right and two to the left of center. The angular distance between the center of the display and the center of the two inside-position letters was $.61 \mathrm{deg}$ and $1.73 \mathrm{deg}$ for the two outside positions. These are the same distances used by Weisstein and Haber (1965). A black fixation $X$ subtending $7.5 \mathrm{~min}$ of visual angle was centered $.6 \mathrm{deg}$ of visual angle below the imaginary line on which the stimuli appeared. To equalize viewing conditions, a transparent plastic card was installed in front of the holder for the letter stimuli and a white card was placed in back of the holder for the transparent cards containing the masking rings. Adjustable card holders made possible perfect alignment of the letters within each of the masking rings.

Procedure. Masking rings were presented at ISIs of $0,10,20,30,40,60,90$, and $120 \mathrm{msec}$ after the letter stimuli. The fixation field was on at all times except during the stimulus flashes. It was decided not to use concurrent presentation since this would involve either presentation of the two stimulus fields simultaneously, with resulting luminance summation between the two fields, or both rings and letters would have to be drawn on the same cards. Neither condition would be directly comparable with conditions of the other delay intervals.

All Ss took part in practice sessions before beginning the experiment. During these sessions it was found that the exposure durations employed by Weisstein and Haber ( $20 \mathrm{msec}$ for the letter and $50 \mathrm{msec}$ for the masking ring) resulted in perfect performance at all delay intervals for our Ss. As a result, separate exposure durations were set for each $S$ to yield approximately $85 \%$ accuracy at 0 ISI. Exposure time for the ring was maintained at two and a half times the duration used for the letter, the ratio used by Weisstein and Haber (1965). Times used ranged from $5 \mathrm{msec}$ for the letter with $121 / 2 \mathrm{msec}$ for the ring, to $10 \mathrm{msec}$ for the letter with $25 \mathrm{msec}$ for the ring.

Each experimental session consisted of presentation of one block of 16 trials at each ISI interval. There were four sessions per S, yielding 64 trials per ISI for each S. The order of presentation of the ISIs within each session were randomized for each S. Eight different random presentation orders for the stimuli were constructed with the restrictions that no stimulus or position appear more than three times in a row. Each of these orders was used once at each delay interval for each $S$. Two of the Ss received trial-by-trial feedback, the other two did not. The 90and 120-msec ISIs were dropped after the first two sessions as Ss were making no errors at these times. Each session was preceded by $5 \mathrm{~min}$ of dark adaptation plus a brief warm-up with feedback at 0 ISI. During the experimental session, $E$ gave a ready signal before each trial, after which the $S$ was to get the fixation $X$ in clear focus before triggering the stimulus onset. The Ss were instructed to respond either $\mathrm{O}$ or D after each trial.

\section{Results}

Percent correct identifications of Os and Ds were analyzed in a three-way analysis of variance (ISIs, inner vs outer position of the target letter, and Ss). Two of the three main effects were significant [ISIs, $F(7,21)=9.78, \quad p<.001$, and Ss, $F(3,21)=6.29, p<.01]$. Although performance was poorer for the outer position, the position effect did not approach significance, nor did any of the interactions. In Fig. 1, percent correct identifications are shown as a function of ISI. The curve represents average performance, and the performance of each of the four Ss is also shown.

These results are quite at variance with those reported by Weisstein and Haber (1965) and Mayzner et al (1965). The function for average performance suggests that masking is essentially uniform over ISI values to $40 \mathrm{msec}$ and then dissipates quite rapidly in the range to $90 \mathrm{msec}$. Since the longest target duration employed for any $S$ was $10 \mathrm{msec}$, the range of apparent uniform masking represented a total temporal duration of $50 \mathrm{msec}$. Another three-way analysis of variance was performed employing only the five ISI values of 0 to $40 \mathrm{msec}$. Ss were again a significant main effect but the ISI variable did not approach significance $[F(4,16)=2.52, p>.10]$, suggesting that the variation in masking performance at ISI values of $40 \mathrm{msec}$ or less did not differ significantly from each other.

Examination of the individual $S$ curves shows considerable variability in performance at ISI values of $40 \mathrm{msec}$ or less. Several Ss do suggest U-shaped masking functions but the failure of the $S$ by ISI interaction to approach significance in either analysis of variance $[F(21,21)=1.74$ and $F(12,12)=1.62$, respectively] indicates that these individual differences are within random error variance of the average performance.

It might be argued that since the trials were presented in blocks of 16 , all at the same ISI value, the Ss had a better chance to adapt to each condition, thus reducing their error rates. However, a check of the data showed that no more errors occurred on the first trial of a block than on the eighth trial, suggesting that such an effect did not take place.

Before considering other implications of the data and possible reasons for discrepancies between our findings and those of Weisstein and Haber (1965), the results from two other experiments will be considered.

\section{EXPERIMENT 2}

There was some suggestion of U-shaped masking function in the individual $S$ data of Experiment 1. Although the $S$ by ISI interactions did not approach significance, this may have been due to too few trials at each ISI value to permit a sensitive 


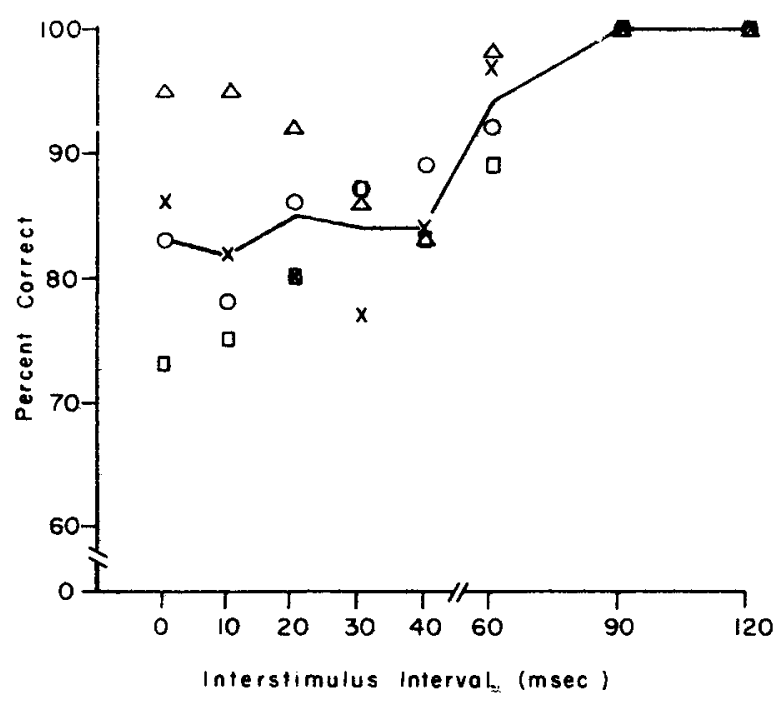

Fig. 1. Percent correct identifications of $O$ and $D$ as a function of ISI. The curve represents average performance, and the data points for the individual Ss are also shown.

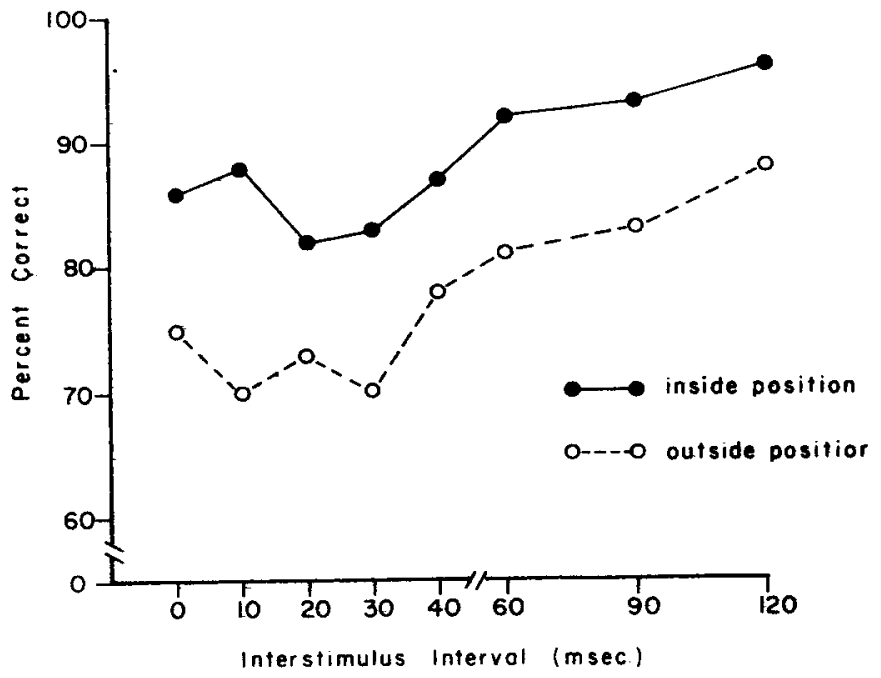

Fig. 2. Percent correct identifications for $H$ and $K$ as a function of ISI and retinal location. assessment of individual differences in masking functions. In the present experiment, the number of observations per $S$ per ISI were doubled (128 trials). Also in Experiment 1, Ss had reported the phenomenal experience of the $O$ seeming to move into the masking ring. Kahneman (1967) has pointed out that the apparent shape of the masking function obtained by Weisstein and Haber (1965) could be attributed to apparent movement due to the shape compatibility of the target letters and masking ring. To remove this possibility, in the present experiment the target letters were $H$ and $K$. They were chosen because their form is not compatible with the masking ring and thus would tend to reduce tendencies toward apparent movement. Further, both letters have their distinguishing characteristics on the periphery as do the $O$ and $D$ and thus should be susceptible to the same type of contour masking effects as the latter letters.

\section{Method}

Subjects. Four undergraduates at the University of Illinois (one male) served as paid volunteers. All but the male $S$ had taken part in Experiment 1.

Apparatus and stimuli. The equipment was the same as that in Experiment 1. The target letters $\mathrm{H}$ and $\mathrm{K}$ were drawn with the same size template and point as the $O$ s and Ds in the previous experiment. They were presented in the same four positions with reference to the fixation point. Masking rings were also the same as in the previous experiment. The letter $\mathrm{H}$ subtended $.62 \mathrm{deg}$ vertically by $.47 \mathrm{deg}$ horizontally. Maximum angular separation between the
$\mathrm{H}$ and the masking rings was $.075 \mathrm{deg}$ on each side. The letter $\mathrm{K}$ subtended $.62 \mathrm{deg}$ vertically by $.45 \mathrm{deg}$ horizontally $(.23 \mathrm{deg}$ at its narrowest point). Thus the maximum angular separation between the $K$ and the ring on the left was $.085 \mathrm{deg}$ and on the right was .195 deg. All other viewing conditions were the same as in Experiment 1.

Procedure. The same ISIs were used as in Experiment 1. The new $\mathrm{S}$ was given a practice session before beginning the experiment. Prior to the experimental sessions, exposure durations were determined for each $S$ that yielded approximately $85 \%$ accuracy at 0 ISI. Since the $\mathrm{H}$ and $\mathrm{K}$ were much more easily discriminated than the $O$ and $D$, a 1.0 neutral density filter was installed in the viewing hood rather than reducing exposure durations. Under these conditions, exposure times ranged from $5 \mathrm{msec}$ for the letter stimuli with $12 \frac{1}{2} \mathrm{msec}$ for the masking ring to $7 \frac{1}{2} \mathrm{msec}$ for the letters and $18.3 \mathrm{msec}$ for the masking ring.

The experimental sessions were conducted in the same manner as in the previous experiment. There were eight sessions per $S$ yielding 128 trials per ISI per S.

\section{Results and Discussion}

Percent correct letter identifications were analyzed in a three-way analysis of variance (ISIs, inner vs outer target position, and Ss). All three main effects were significant $(p<.01)$. Of particular interest was the failure of the $S$ by ISI interaction to approach significance $[F(21,21)=.83, p>.10]$

The results are shown in Fig. 2, where percent correct identifications are plotted as a function of inner vs outer target position and ISI. There is an appreciable difference in identification of the targets as a function of the target location. This result is consistent with Experiment 1 and is commensurate with the reduction in acuity for targets further removed from the center of the fovea. The inner targets were $.61 \mathrm{deg}$ from the fixation point as opposed to the location of the outer letters at $1.73 \mathrm{deg}$ from fixation. There is a slight suggestion of a U-shaped masking function for targets located on the inner position. The minimum value of the function falls at an ISI of $20 \mathrm{msec}$. That this is to be attributed to chance, however, is shown by the failure of the Positions by ISI interaction to approach significance $[F(7,21)=1.04, \quad p>.10]$. Further evidence that the variability in the fucntions for inner and outer positions are attributable to chance is shown by an analysis of variance for only those ISI values of 0 - to $40-\mathrm{msec}$ ISI. The effect of ISI on this restricted range does not approach significance $[\mathrm{F}(4,12)=1.29$, $\mathrm{p}>.10]$, suggesting as did the previous experiment that masking is relatively uniform over a total temporal range of $50 \mathrm{msec}$.

Although the number of observations per S per ISI was doubled over that of Experiment 1, there was still no suggestion of reliable individual differences in masking functions $[F(21,21)=.83, p>.20]$. Thus, the variation between Ss in masking at values less than 40-msec ISI would appear to be attributable to the random error that is inherent in a task having a $50 \%$ chance rate and where performance is set at less 
than $100 \%$ by manipulation of the stimulus intensity.

\section{EXPERIMENT 3}

Although the results obtained so far would seem quite conclusive, one final check on their validity was carried out. A forward masking paradigm was employed using the letters $H$ and $K$ from Experiment 2. Weisstein's (1968) theory of masking would not predict a $U$ shape for the forward masking arrangement. While the above results have suggested that the individual differences observed in masking functions were well within error expectations, nonetheless, if the same suggestions of $U$ shapes were obtained for individual $S s$ under a forward masking paradigm, our confidence would be increased that those observed in the previous experiment were attributable to random error.

\section{Method}

Subjects. Three female undergraduates at the University of Illinois served as paid volunteers. None had participated in either of the two previous experiments and all had normal vision.

Apparatus and stimuli. These were the same as those employed in Experiment 2.

Procedure. Prior to beginning experimental sessions, each $S$ was given two practice sessions during which she was presented with the ring followed at 0 ISI by one of the target letters. In addition to acquainting $S$ with the task, these sessions served to determine exposure durations that under the 0 ISI condition led to identification performance of approximately $70 \%$. These values were then employed in the experimental sessions. The average exposure duration of the target was $9.3 \mathrm{msec}$ and the ring duration was twice the target duration. Following the practice sessions, each $\mathbf{S}$ served in eight experimental sessions. Each session consisted of a presentation of the masking ring followed at one of eight ISIs $(0,10,20,30,40,50,60$, and $90 \mathrm{msec})$ by one of the target letters in one of the four possible positions. Order of presentations of ISIs within sessions was counterbalanced across sessions and randomized across Ss. Each letter and ring combination was presented in the four positions an equal number of times at all ISI values and its occurrence was counterbalanced. As in the preceding experiments, Ss were dark adapted for approximately $10 \mathrm{~min}$ before beginning an experimental session and luminances of the fields were constantly monitored by a MacBeth illuminometer and a Spectra brightness spot meter.

\section{Results}

Percent correct identifications were again analyzed in a three-way classification analysis of variance (ISIs, inner vs outer target position, and Ss). The effect of ISI was again significant $[F(7,14)=3.01$, $p<.05]$ as was also the $S$ variable $[F(2,14)=9.20, p<.01]$. The position effect was not significant $[\mathrm{F}(1,2)=12.14$, $p>.05]$ when evaluated against the significant $S$ by Position effect $[F(2,14)=7.81, p<.01]$. Again the $S$ by ISI interaction failed to approach significance $[F(14,14)=2.05, p>.05]$.

In Fig. 3, percent correct identifications of the $\mathrm{H}$ and $\mathrm{K}$ are shown as a function of ISI for each of the three Ss. The results are very similar to those obtained in Experiments 1 and 2 employing the backward masking paradigm. Again there are individual differences, but the overall trend is for essentially uniform masking over the range of ISIs to 30 or $40 \mathrm{msec}$.

The individual $S$ variability is of particular interest. Weisstein's (1968) theory of masking in terms of differential latency for inhibitory and excitatory processes would not account for U-shaped masking functions under a forward masking paradigm. It is to be noted that there is as much suggestion of U-shaped masking functions for $S s \quad 1$ and 3 in the forward masking paradigm of the present experiment as there was in the individual $S$ curves in Experiments 1 and 2. This would reinforce the conclusion suggested by the lack of significant $S$ by ISI effects that what variation is obtained between $S$ s in the masking region can be attributed to random error components.

Any two-alternative forced-choice task is bound to have a large random error component when stimulus energies are adjusted to a level that insures less than $100 \%$ performance. If energy is adjusted to a performance criterion of $80 \%$ correct, the best estimate of the proportion of the correct responses that are due to pure chance or guessing factors is $20 \%$. This means that $\mathrm{S}$ is guessing on $40 \%$ of the trials with the attendant variation in performance that this is going to entail.

\section{GENERAL DISCUSSION}

In none of the three experiments do we find any confirmation or support for the U-shaped masking functions reported by Weisstein and Haber (1965). Instead, our results show that under these experimental arrangements the masking function obtained with either a backward or forward paradigm is best characterized as a monotonic function of ISI

Our failure to replicate the Weisstein and Haber (1965) results could be due to a number of factors. First of all, we were disturbed by our inability to obtain masking in our $S$ population under the precise exposure durations reported by

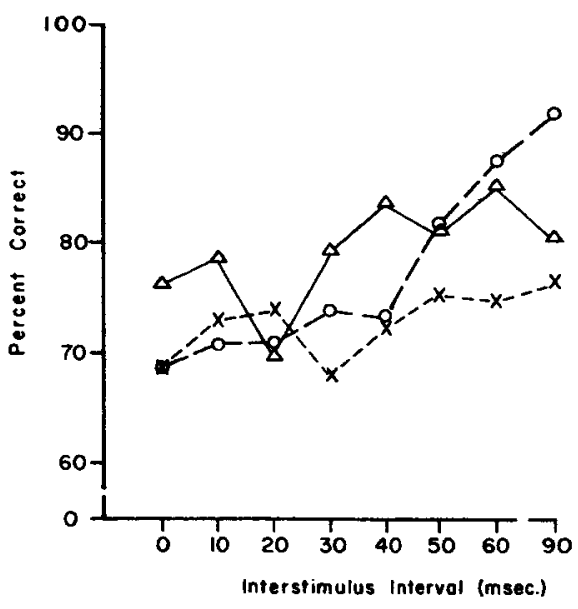

Fig. 3. Percent correct identifications for $H$ and $K$ as a function of ISI for individual Ss under a forward masking paradigm.

Weisstein and Haber (1965). They used a uniform 20-msec exposure of the $O$ or $D$ and a 50-msec duration for the mask for all Ss. Employing the same luminance levels and apparently the same contrast of stimuli as they did, we found that in our $S$ population no masking at all was obtained with these exposure durations. We had to reduce the exposure duration of the target letters on the average more than half in order to obtain a masking effect. The mask duration was also correspondingly reduced in order to maintain the same energy ratio between target and mask as employed by Weisstein and Haber (1965).

We are also puzzled by their ability to obtain Ss who show a comparable performance at a constant exposure duration. We had to adjust individually the exposure duration of the target to compensate for individual differences in perceptual recognition performance at brief exposure durations. In many years of tachistoscopic experimentation, we always have found it necessary to use an individually tailored exposure duration. Attempts to employ a constant exposure duration for all Ss invariably leads to a large proportion of the Ss who find the task too easy under all experimental conditions and, therefore, give $100 \%$ performances under all conditions. Another large proportion of Ss find the task so difficult as to yield just chance performance under all exposure conditions. If a large enough sample of Ss is employed, there will be $\mathrm{Ss}$ in the middle range who will find the constant exposure duration approximately appropriate for their capabilities and who will, therefore, reveal experimental effects. However, such a methodology is extremely inefficient, since approximately half of the Ss contribute nothing to the sensitivity of the 
experiment, and the generalization of the results is limited to $S s$ whose perceptual capability was in the vicinity of the arbitrarily chosen exposure duration. Weisstein and Haber (1965) do not say whether or not a number of Ss had to be screened in order to find the four Ss whose perceptual capabilities corresponded with the chosen exposure duration of $20 \mathrm{msec}$.

A methodological advantage of adjusting the target exposure duration to the capability of the individual $S$ is that it permits a generalization of the results to a larger sample of people. By using Ss as a variable in the analysis of variance, the interaction of Ss by experimental conditions and treatments can be evaluated. Thus, it is possible to determine if there are individual differences in the functional relationships obtained.

A second difference between our experiment and that of Weisstein and Haber (1965) was the lack of a concurrent target and mask presentation in our studies. We did not employ this condition, since it is extremely difficult, if not impossible, to make a concurrent presentation of target and mask directly comparable to the other ISI values. If the two fields of the tachistoscope are used to superimpose the target and mask simultaneously, one encounters the $\mathrm{p} \mathrm{roblem}$ of $1 \mathrm{u} \mathrm{minance}$ summation-contrast reduction for both the target and the mask (Eriksen, 1966). This contrast reduction will be different from that obtained at any other ISI value, even though an illuminated ISI field is employed. Neither Weisstein and Haber (1965) nor Mayzner et al (1965) refer to how they solved this problem, but one might infer that the technique employed was that used by Weisstein (1966) in a succeeding experiment. Here the target and masking ring were drawn on the same stimulus card and exposed in only one field of a tachistoscope. This, however, poses even more serious restrictions. Since they are on the same card, the exposure duration of both the target and the masking ring have to be identical. This would mean that both were exposed for $20 \mathrm{msec}$, whereas in all other ISI conditions the target was exposed for $20 \mathrm{msec}$ but the mask had a $50-\mathrm{msec}$ duration. It is not at all surprising that less masking would be obtained in the concurrent presentation where the mask has only $40 \%$ as much energy as it has at all other ISI values.

A third possibility that may account for discrepancies between Weisstein and Haber's results and ours has to do with the alignment of the target letters and masking rings. The inner diameter of the masking ring is almost the same as the outer diameter of the target letters, with the result that slight misalignments can result in target and masking ring overlap. This would be of critical importance if it occurred on the side that distinguishes the $D$ from the $O$. We found it an extremely time-consuming task to construct the stimulus cards so as to achieve perfect alignment. Weisstein and Haber (1965) do not describe their method employed to assure alignment, but in a succeeding paper Weisstein (1966) describes automatic equipment that positioned target and masks in the two separate fields. The essentials of the equipment were the drawing of the targets on one roll of paper and the masking rings on another roll of paper that were then mounted on rollers that were advanced automatically in the target and masking fields of the tachistoscope. It is difficult to appreciate how precise alignment could be achieved in this manner. Given the lack of rigidity of rolled paper and the problems of building gear systems that are precise enough to assure that the targets are going to be precisely aligned in the center of the mask, this automatic system would constitute an impressive engineering feat. If only one set or roll of targets and masks were employed, then any systematic misalignment would be systematic through all Ss and could possibly account for the U-shaped masking functions obtained.

Results of the three experiments are consistent with an explanation of visual masking in terms of lack of fine temporal resolution in the visual system. Due to this lack of resolution, successive stimuli separated by short enough temporal intervals are essentially stacked into a composite stimulus, with degree of this summation or integration inversely proportional to the temporal duration over which the successive stimuli are distributed. The facts of this temporal integration in vision are exceedingly well documented, varying from the well known Bloch's law to recent work demonstrating temporal integration in form identification tasks (Kahneman, 1964; Kahneman \& Norman, 1964; Schurman, Eriksen, \& Rohrbaugh, 1968). Indeed, if the masking paradigm did not lead to a summation of the target and masking stimulus into a composite at short ISIs the basic question to be asked would be, why not?

The integration of form information over time in vision can be readily demonstrated. If a pattern of alternate black and white stripes is presented in one field of a tachistoscope in a vertical alignment, and a second stimulus field presents the same pattern but in a horizontal alignment and the two patterns are flashed successively, the phenomenal experience is that of a checkered pattern. The checkered pattern can be seen over
ISIs as long as $100 \mathrm{msec}$ or more depending upon the luminance and durations of the two stimulations.

If the existence of temporal summation demonstrated in this manner is not satisfactory, Eriksen and Collins (1967, 1968) have used a similar procedure to experimentally demonstrate temporal integration. Their stimuli were two patterns, each by itself appearing to be a random pattern of dots, but when the two patterns were superimposed, one in each field of a tachistoscope, a nonsense syllable could be read. When each dot pattern was presented for $5 \mathrm{msec}$ and the interval between the successive presentations was varied, evidence of integration of the dot pattern was obtained over intervals in excess of $100 \mathrm{msec}$.

It is to be noted that these experiments are essentially the same as a backward or forward masking paradigm. The difference is that in the typical masking paradigm a summation or integration of the target and masking stimulus is expected to impair target identification. In the Eriksen and Collins studies, however, if the composite occurs, then it is possible for the $S$ to identify the nonsense syllable. If masking was due to an inhibition of the first stimulus by the second or by some erasure process (Averbach \& Coriell, 1961; Sperling, 1963), then the second dot pattern should have served to erase or inhibit the first dot pattern and no integration should have occurred from which the nonsense syllable could be perceived.

Uttal (1969) has also recently employed dot characters embedded in preceding and following random noise dot patterns. As would be expected from the present results and from an integration theory of masking, performance is a monotonic function of the temporal separation of the character and the preceding or following random dot masking patterns.

It is to be noted that Weisstein's (1968) theory of masking is not incompatible with the temporal integration account of the phenomena. Her postulation of a difference in latency between inhibitory and excitatory cells could well account for discrepancies in monotonic functions that might be found. Thus, if U-shaped masking functions should be substantiated under certain special circumstances, Weisstein's (1968) differential latencies for excitatory and inhibitory cells might well be the means of reconciling such possible U-shaped functions with an overall temporal integration theory of masking. However, the existence of a latency difference between inhibitory and excitatory cells would seem to be more readily substantiated by neurophysiological techniques than by further resort to data at 
the psychophysical level. By means of single cell recording techniques it should be possible to examine the latency of response of a population of inhibitory cells and a population of excitatory cells and determine if there is indeed a latency difference.

The above discussion holds only with respect to detection or discriminatory responses under the masking paradigm. There's little question but that a U-shaped masking function is obtained if $\mathrm{Ss}$ are asked to judge or rate the apparent brightness of the target stimulus. In terms of phenomenal brightness, the target becomes progressively less bright as ISI increases up to some value (depending upon the luminances and nature of the target and masking stimuli) and then becomes progressively brighter as ISI increases. This effect was noted by all Ss in the present experiment, but as the data show, the phenomenal dimming apparently is an independent or a parallel effect to the identification process. The information necessary for detection or identification does not seem to be affected by the phenomenal dimming. As noted previously, this result is analogous to the finding of Eriksen and Colegate (1969) that apparent movement of forms does not impair their identifiability.

The phenomenal dimming of a target stimulus is as yet inadequately explained, but it is likely that it is one facet of the overall temporal integration process in vision. It may be that it reflects the diminishing contribution of the first stimulus to the composite as ISI increases beyond the range of complete summation and enters the range where the summation is only partial.

\section{REFERENCES}

AVERBACH, E., \& CORIELL, A. S. Short-term memory in vision. Bell System Technical Journal, 1961, 40, 309-328.

ALPERN, M. Metacontrast. Journal of the Optical Society of America, 1953, 43, 648-657.

ERIKSEN，C. W. Temporal luminance summation effects in backward and forward masking. Perception \& Psychophysics, 1966, 1, 87-92.

ERIKSEN, C. W., \& COLEGATE, R. L. Identification of forms at brief durations when seen in apparent motion. Journal of Experimental Psychology, in press.

ERIKSEN, C. W., \& COLLINS, J. F. Some temporal characteristics of visual pattern perception. Journal of Experimental Psychology, 1967, 74, 476-484.

ERIKSEN, C. W., \& COLLINS, J. F. Sensory traces versus the psychological moment in the temporal organization of form. Journal of Experimental Psychology, 1968, 77, 376-382.

ERIKSEN, C. W., \& MARSHALL, P. H. Failure to replicate a reported $\mathrm{U}$-shaped visual masking function. Psychonomic Science, 1969, 15, 195-196.

FEHRER, E., \& SMITH, E. Effect of luminance ratio on masking. Perceptual \& Motor Skills, $1962,14,243-253$.

FLOM, M. C., WEYMOUTH, F. W., \& KAHNEMAN, D. Visual resolution and contour interaction. Journal of the Optical Society of America, 1963, 53, 1026-1032.

KAHNEMAN, D. Temporal summation in an acuity task at different energy levels $-A$ study of the determinants of summation. Vision Research, 1964, 4, 557-566.

KAHNEMAN, D. An onset-onset law for one case of apparent motion and metacontrast. Perception \& Psychophysics, 1967, 2, 577-584.

KAHNEMAN, D. Method, findings, and theory in studies of visual masking. Psychological Bulletin, 1968, 70, 404-425.

KAHNEMAN, D., \& NORMAN, J. The time-intensity relation in visual perception as a function of observer's task. Journal of Experimental Psychology, 1964, 68, 215-220.

MAYZNER, M. H., BLATT, M. H., BUCHSBAUM, W. H., FRIEDEL, R. T., GOODWIN, P. E., KANON, D., KELEMAN, A., \& NILSSON, W. D. A U-shaped backward masking function in vision. A partial replication of the Weisstein and Haber study with two ring sizes. Psychonomic Science,
$1965,3,79-80$.

SCHILLER, P. H., \& SMITH, M. C. Detection in metacontrast. Journal of Experimental Psychology, 1966, 71, 32-39.

SCHURMAN, D. L. Dark intervals as stimulus events and their effect on visual masking and time-intensity reciprocity. Unpublished doctoral dissertation, Unviersity of Illinois, 1970.

SCHURMAN, D. L., \& ERIKSEN, C. W. Summation and interaction of successive masking stimuli in visual perception. American Journal of Psychology, 1969, 82, 320-332.

SCHURMAN, D. L., ERIKSEN, C. W., \& ROHRBAUGH, J. Masking phenomena and time intensity reciprocity for form. Journal of Experimental Psychology, in press.

SPERLING, G. A model for visual memory tasks. Human Factors, 1963, 5, 19-31.

STIGLER, R. Chronophotische Studien über den Umgebungskontrast. Pflügers Archiv für die gesamte Physiologie des Menschen und die Tiere, $1910,134,365-435$.

UTTAL, W. $R$. The character in the hole experiment. Interaction of forward and backward masking of alphabetic character recognition by dynamic visual noise (DVN). Perception \& Psychophysics, 1969, 6. 177-181.

WEISSTEIN, N. Backward masking and models of perceptual processing. Journal of Fxperimental Psychology, 1966, 72, 232-240.

WEISSTEIN, N. A Rashevsky-Landahl neural net Simulation of metacontrast. Psychological Review, 1968, 75, 494-521.

WEISSTEIN, N., \& HABER, R. N. A U-shaped backward masking function in vision. Psychonomic Science, 1965, 2, 75-76.

\section{NOTES}

1. This investigation was supported by Public Health Service Research Grant MH-1206 and a Public Health Service Research Career Program award, K6-MH-22,014.

2. Address: University of Illinois, Urbana. Illinois 61801 .

3. Personal communication from Peter $\mathbf{H}$ Schiller.

(Accepted for publication January 7, 1970.) 